HUTP-97/A021

\title{
Inclusive Decay Distributions of Coherent Two-body States
}

\author{
Hitoshi Yamamoto \\ Dept. of Physics, Harvard University, 42 Oxford St., Cambridge, MA 02138, U.S.A.
}

(September 16, 2018)

\begin{abstract}
When a vector meson such as $\phi, J / \Psi(3 S)$ or $\Upsilon(4 S)$ decays to a particleantiparticle pair of neutral mesons, the time distribution of inclusive decay to a given final state is naively expected to be the incoherent sum of those of the two mesons with opposite flavors. In this paper, we show that this is in general not the case for arbitrary coherent two-body states of the mesons, and obtain conditions under which such a naive incoherent sum gives the correct distributions. The analysis is based on the Weisskopf-Wigner formalism, and applicable to the cases where there are more than two orthogonal states that can mix to form a set of eigenstates of mass and decay rate.

03.65.-w, 13.20.Gd, 13.25.He
\end{abstract}

Typeset using REVTEX 


\section{INTRODUCTION}

In the studies of $C P$ violation in neutral meson systems such as $\phi \rightarrow K^{0} \bar{K}^{0}$ []] $J / \Psi(3 S) \rightarrow D^{0} \bar{D}^{0}$, or $\Upsilon(4 S) \rightarrow B^{0} \bar{B}^{0}$ [2], one often deals with an inclusive decay distribution where one of the mesons decay to a given final state $f$ at a given time $t$ and the other meson can decay to any final state at any time. It has been shown in a recent study that in the decay of $\Upsilon(4 S)$, the inclusive decay time distribution of $\Upsilon(4 S) \rightarrow f$ is the incoherent sum of the decay time distribution of a pure $B^{0}$ at $t=0$ decaying to $f$ at time $t$ (denoted $\left.\Gamma_{B^{0} \rightarrow f}(t)\right)$ and that of $\bar{B}^{0}$ (denoted $\left.\Gamma_{\bar{B}^{0} \rightarrow f}(t)\right)$ [3]. Such relation is critical in analyses of inclusive lepton asymmetries [3], or in relating observed inclusive yield asymmetries of $\Upsilon(4 S) \rightarrow f$ and $\Upsilon(4 S) \rightarrow \bar{f}$ to the asymmetry of decay amplitudes $A m p\left(\bar{B}^{0} \rightarrow f\right)$ and $\operatorname{Amp}\left(B^{0} \rightarrow \bar{f}\right)$ 团. When a $\Upsilon(4 S)$ decays to a pair of neutral $B$ mesons, it is generated in a coherent $L=1$ state, which is antisymmetric under the exchange of the two mesons:

$$
\frac{1}{\sqrt{2}}\left(\left|\bar{B}^{0}(\vec{k})\right\rangle\left|B^{0}(-\vec{k})\right\rangle-\left|B^{0}(\vec{k})\right\rangle\left|\vec{B}^{0}(-\vec{k})\right\rangle\right)
$$

where the mesons are labeled by their momentum $\pm \vec{k}$ which we will drop hereafter and implicitly assume that left (right) side of the meson pair is in $+\vec{k}(-\vec{k})$ direction. A natural question is then whether such an incoherent sum gives the correct inclusive distribution for a general two-body state given by

$$
\Psi=a B^{0} B^{0}+b B^{0} \bar{B}^{0}+c \bar{B}^{0} B^{0}+d \bar{B}^{0} \bar{B}^{0},
$$

where $a, b, c, d$ are arbitrary complex coefficients with

$$
|a|^{2}+|b|^{2}+|c|^{2}+|d|^{2}=1 .
$$

When the pair is generated with a definite orbital angular momentum, further symmetry relations must be satisfied; in this study, however, we will keep the general form as above. As we will show below, the necessary and sufficient condition for the naive incoherent sum

$$
\begin{aligned}
\left(2|a|^{2}+\right. & \left.|b|^{2}+|c|^{2}\right) \Gamma_{B^{0} \rightarrow f}(t) \\
& +\left(2|d|^{2}+|b|^{2}+|c|^{2}\right) \Gamma_{\bar{B}^{0} \rightarrow f}(t)
\end{aligned}
$$


to give the correct distribution for any final state $f$ (and independent of the details of the mixing) is

$$
G \stackrel{\text { def }}{\equiv} a^{*}(b+c)+d(b+c)^{*}=0 .
$$

\section{GENERAL COHERENT TWO-BODY STATES}

In the following, we study a system of $n$ orthogonal states $B_{i}(i=1, \ldots, n)$ mixing to form $n$ eigenstates of mass and decay rate (physical states) $B_{\alpha}(\alpha=a, b, c \ldots)$. The eigenstates $B_{\alpha}$ are not necessarily orthogonal when $C P$ is violated. We use the WeisskopfWigner formalism [5], but no assumptions are made on $C P$ or $C P T$ symmetries unless otherwise stated. The essential approximation used in the formalism is that the oscillations caused by mass differences and the decay rates are sufficiently slower than the time scale of decay transitions, which is a very good assumption for the cases under study [6].

The eigenstates $B_{\alpha}$ then evolve as

$$
B_{\alpha} \rightarrow e_{\alpha}(t) B_{\alpha}, \quad e_{\alpha}(t) \stackrel{\text { def }}{\equiv} e^{-\left(\gamma_{\alpha} / 2+i m_{\alpha}\right) t},
$$

where $\gamma_{\alpha}$ and $m_{\alpha}$ are the decay rate and mass of the physical state $B_{\alpha}$. The time $t$ is the proper time of the particle under consideration. The Weisskopf-Wigner formalism can be relativistically extended to moving particles; it can be shown, however, that it is equivalent to the evolution in the rest frame formulated as above [7]. We will hereafter consistently use

the indices $i, j$ for the orthogonal states $B_{1}, B_{2} \ldots$, and Greek indexes $\alpha, \beta$ for the physical states $B_{a}, B_{b} \ldots$ :

$$
\begin{aligned}
& \text { Orthogoanl states: } i, j, i^{\prime}, j^{\prime}=1,2 \ldots n \\
& \text { Physical states: } \alpha, \beta, \alpha^{\prime}, \beta^{\prime}=a, b \ldots(n \text { total }) .
\end{aligned}
$$

The eigenstates $B_{i}$ can be written as linear combination of $B_{\alpha}$ 's:

$$
B_{i}=\sum_{\alpha} r_{i \alpha} B_{\alpha}
$$


For the system composed of $B^{0}$ and $\bar{B}^{0}$, we have $n=2$ :

$$
B_{1}=B^{0}, \quad B_{2}=\bar{B}^{0}
$$

and the physical states are usually written as

$$
\left\{\begin{array}{l}
B_{a}=p B^{0}+q \bar{B}^{0} \\
B_{b}=p^{\prime} B^{0}-q^{\prime} \bar{B}^{0}
\end{array}\right.
$$

or solving for $B^{0}$ and $\bar{B}^{0}$,

$$
\left\{\begin{array}{l}
B^{0}=c\left(q^{\prime} B_{a}+q B_{b}\right) \\
\bar{B}^{0}=c\left(p^{\prime} B_{a}-p B_{b}\right)
\end{array}\right.
$$

with

$$
c \stackrel{\text { def }}{=} \frac{1}{p^{\prime} q+p q^{\prime}} ;
$$

namely,

$$
\begin{aligned}
& r_{1 a}=c q^{\prime}, r_{1 b}=c q, \\
& r_{2 a}=c p^{\prime}, r_{2 b}=-c p
\end{aligned}
$$

Returning to the general case of $n$ orthogonal states, the orthonormality of $B_{i}$ 's can be expressed in terms of the physical eigenstates $B_{\alpha}$ as

$$
\delta_{i j}=\left\langle B_{i} \mid B_{j}\right\rangle=\sum_{\alpha \beta} r_{i \alpha}^{*} r_{j \beta}\left\langle B_{\alpha} \mid B_{\beta}\right\rangle
$$

where we have used (2.2). The decay amplitude of a pure $B_{i}$ state at $t=0$ decaying to a final state $f$ at time $t$ is, from (2.2) and (2.1),

$$
A_{B_{i} \rightarrow f}(t)=\sum_{\alpha} r_{i \alpha} a_{\alpha f} e_{\alpha}(t)
$$

where $a_{\alpha f}$ is the amplitude of $B_{\alpha}$ decaying to $f$ :

$$
a_{\alpha f} \stackrel{\text { def }}{\equiv} A m p\left(B_{\alpha} \rightarrow f\right)
$$

The normalization is such that $\left|a_{\alpha f}\right|^{2}$ is the partial decay rate of $B_{\alpha}$ to $f$ : 


$$
\sum_{f}\left|a_{\alpha f}\right|^{2}=\gamma_{\alpha} .
$$

Namely, the density of the final states, more precisely the square root of it, is absorbed into the definition of the amplitude.

The time dependent decay amplitudes $A_{B_{i} \rightarrow f}(t)$ satisfy the following orthonormality relation [10], where the 'inner product' of $A_{B_{i} \rightarrow f}$ and $A_{B_{j} \rightarrow f}$ is defined by integration of $A_{B_{i} \rightarrow f}^{*} A_{B_{j} \rightarrow f}$ over time followed by summation over all possible final states:

$$
\begin{aligned}
\sum_{f} \int_{0}^{\infty} d t & A_{B_{i} \rightarrow f}^{*}(t) A_{B_{j} \rightarrow f}(t) \\
& =\sum_{\alpha \beta} r_{i \alpha}^{*} r_{j \beta} \sum_{f} a_{\alpha f}^{*} a_{\beta f} \int_{0}^{\infty} d t e_{\alpha}^{*}(t) e_{\beta}(t) \\
& =\sum_{\alpha \beta} r_{i \alpha}^{*} r_{j \beta} \frac{\sum_{f} a_{\alpha f}^{*} a_{\beta f}}{\frac{\gamma_{\alpha}+\gamma_{\beta}}{2}-i\left(m_{\alpha}-m_{\beta}\right)} \\
& =\delta_{i j} .
\end{aligned}
$$

In deriving the above, where we have used the generalized Bell-Steinberger relation [8] given by

$$
\frac{\sum_{f} a_{\alpha f}^{*} a_{\beta f}}{\frac{\gamma_{\alpha}+\gamma_{\beta}}{2}-i\left(m_{\alpha}-m_{\beta}\right)}=\left\langle B_{\alpha} \mid B_{\beta}\right\rangle,
$$

together with the orthonormality of $B_{i}$ 's (2.5). Note that the relation (2.8) reduces to the amplitude normalization condition (2.6) for $\alpha=\beta$. While the Bell-Steinberger relation can be derived by requiring that unitarity is satisfied [8], it can also be derived from the old-fashioned perturbation theory to the lowest non-trivial order [9]. The probability that a pure $B_{i}$ at $t=0$ decays to a final state $f$ at time $t$ is simply the square of the time-dependent amplitude:

$$
\Gamma_{B_{i} \rightarrow f}(t)=\left|A_{B_{i} \rightarrow f}(t)\right|^{2} .
$$

The relation (2.7) with $i=j$ shows that this decay distribution conserves probability:

$$
\sum_{f} \int_{0}^{\infty} d t \Gamma_{B_{i} \rightarrow f}(t)=1 .
$$

Now, take a general coherent two-body state at $t=0$ given by 


$$
\Psi(t=0)=\sum_{i j} c_{i j} B_{i} B_{j}
$$

where $c_{i j}$ are arbitrary complex coefficients with

$$
\sum_{i j}\left|c_{i j}\right|^{2}=1
$$

For simplicity, we will hereafter label the left and right sides of the particle pair as north $(\mathrm{N})$ and south $(\mathrm{S})$. The specific names to distinguish the two sides are irrelevant; we just need some labels for the two orthogonal spaces. The probability that north side decays to a final state $f_{N}$ at time $t_{N}$ and the south side to a final state $f_{S}$ at time $t_{S}$ is then

$$
\Gamma_{\Psi \rightarrow f_{N} f_{S}}\left(t_{N}, t_{S}\right)=\left|\sum_{i j} c_{i j} A_{B_{i} \rightarrow f_{N}}\left(t_{N}\right) A_{B_{j} \rightarrow f_{S}}\left(t_{S}\right)\right|^{2}
$$

From the orthonormality of the decay amplitude (2.7), one sees that this double-time decay distribution also conserves probability; namely, when integrated over the two decay times and summed over all possible final states, it becomes unity:

$$
\sum_{f_{N} f_{S}} \int_{0}^{\infty} d t_{N} \int_{0}^{\infty} d t_{S} \Gamma_{\Psi \rightarrow f_{N} f_{S}}\left(t_{N}, t_{S}\right)=1
$$

We now define the inclusive decay distribution of $\Psi$ to a final state $f$, where $f$ can come from either side of the decay:

$$
\begin{aligned}
\Gamma_{\Psi \rightarrow f}(t) \stackrel{\text { def }}{\equiv} \sum_{f_{N}} \int_{0}^{\infty} d t_{N} \Gamma_{\Psi \rightarrow f_{N} f}\left(t_{N}, t\right) \\
+\sum_{f_{S}} \int_{0}^{\infty} d t_{S} \Gamma_{\Psi \rightarrow f f_{S}}\left(t, t_{S}\right),
\end{aligned}
$$

which, due to (2.10), satisfies

$$
\sum_{f} \int_{0}^{\infty} d t \Gamma_{\Psi \rightarrow f}(t)=2 .
$$

The number 2 comes from the fact that the final state $f$ can come from either side of the decay. The question is under what condition this is equal to the naive incoherent sum

$$
\begin{aligned}
\Gamma_{\Psi \rightarrow f}^{\text {naive }}(t) & \stackrel{\text { def }}{\equiv} \sum_{i j}\left|c_{i j}\right|^{2}\left(\Gamma_{B_{i} \rightarrow f}(t)+\Gamma_{B_{j} \rightarrow f}(t)\right) \\
& =\sum_{i j}\left(\left|c_{i j}\right|^{2}+\left|c_{j i}\right|^{2}\right) \Gamma_{B_{i} \rightarrow f}(t)
\end{aligned}
$$


which is the generalization of (1.3). Using the expression of the double-time distribution (2.9) and the orthonormality of the decay amplitude (2.7), the inclusive decay distribution (2.11) becomes

$$
\begin{aligned}
\Gamma_{\Psi \rightarrow f}(t) & =\sum_{i i^{\prime} j}\left(c_{i j}^{*} c_{i^{\prime} j}+c_{j i}^{*} c_{j i^{\prime}}\right) A_{B_{i} \rightarrow f}^{*}(t) A_{B_{i^{\prime}} \rightarrow f}(t) \\
= & \Gamma_{\Psi \rightarrow f}^{\text {naive }}(t) \\
+ & \sum_{i \neq i^{\prime}} \sum_{j}\left(c_{i j}^{*} c_{i^{\prime} j}+c_{j i}^{*} c_{j i^{\prime}}\right) A_{B_{i} \rightarrow f}^{*}(t) A_{B_{i^{\prime}} \rightarrow f}(t) .
\end{aligned}
$$

The necessary and sufficient condition for this to be equal to $\Gamma_{\Psi \rightarrow f}^{\text {naive }}(t)$ is then

$$
\sum_{i \neq i^{\prime}} G_{i i^{\prime}} A_{B_{i} \rightarrow f}^{*}(t) A_{B_{i^{\prime}} \rightarrow f}(t)=0
$$

with

$$
G_{i i^{\prime}} \stackrel{\text { def }}{=} \sum_{j}\left(c_{i j}^{*} c_{i^{\prime} j}+c_{j i}^{*} c_{j i^{\prime}}\right)
$$

A sufficient condition for (2.12) to be satisfied is clearly

$$
G_{i i^{\prime}}=0 \quad\left(\text { for all } i \neq i^{\prime}\right)
$$

The matrix $G_{i i^{\prime}}$ is 'hermitian' in the sense that

$$
G_{i i^{\prime}}=G_{i^{\prime} i}^{*}
$$

which guarantees that $\Gamma_{\Psi \rightarrow f}(t)$ is a real quantity. Note also that the norm of $G_{i i^{\prime}}$ is re-phase invariant; namely, when the phase of $B_{i}$ 's are re-defined, $G_{i i^{\prime}}$ simply changes its phase:

$$
B_{i} \rightarrow B_{i} e^{i \phi_{i}} \quad \longrightarrow \quad G_{i i^{\prime}} \rightarrow G_{i i^{\prime}} e^{i\left(\phi_{i}-\phi_{i^{\prime}}\right)}
$$

Thus, the condition (2.14) is re-phase invariant.

In the case of $n=2$, the condition (2.14) becomes Eq. (1.4) with $G=G_{12}$. In this case, it is straightforward to show that the condition $G=0$ is the necessary as well as sufficient condition as long as $\gamma_{a} \neq \gamma_{b}, m_{a} \neq m_{b}$, and coefficients $p, q, p^{\prime}, q^{\prime}$ are all non-zero. We still require that $\Gamma_{\Psi \rightarrow f}^{\text {naive }}(t)$ is correct independent of decay amplitudes $a_{\alpha f}$. The proof for general 
case is given in the appendix. The derivation is simple if CPT is conserved in the mixing; namely, $p^{\prime}=p$ and $q^{\prime}=q$. Then the condition (2.12) becomes

$$
\begin{aligned}
& \Re\left(G p q^{*}\right)\left(e^{-\gamma_{a} t}\left|a_{a f}\right|^{2}-e^{-\gamma_{b} t}\left|a_{b f}\right|^{2}\right) \\
& +2 \Im\left(G p q^{*}\right) \Im\left(e^{-\left(\gamma_{+}-i \delta m\right) t} a_{a f}^{*} a_{b f}\right)=0,
\end{aligned}
$$

with

$$
\gamma_{+} \equiv \frac{\gamma_{a}+\gamma_{b}}{2}, \quad \delta m \equiv m_{a}-m_{b}
$$

The three terms in (2.15) have different time dependences, and thus each term should be separately zero. When $\gamma_{a} \neq \gamma_{b}$, this condition is equivalent to $\Re\left(G p q^{*}\right)=\Im\left(G p q^{*}\right)=0$, or simply

$$
G p q^{*}=0 \quad(C P T)
$$

Thus, if both $p$ and $q$ are non-zero (i.e. there is a mixing), $G$ must be zero in order for the naive incoherent sum to be correct.

Let's briefly appreciate the meaning of the condition $G=0$. This is satisfied by any coherent state of $B^{0} \bar{B}^{0}$ and $\bar{B}^{0} B^{0}$ since then we have $a=d=0$. It includes the $\Upsilon(4 S)$ case given by (1.1), or a $B^{0} \bar{B}^{0}$ state with an even orbital angular momentum. Also, the condition is satisfied if $b+c=0$ regardless of the values of $a$ and $d$. However, the condition is not satisfied, for example, by the symmetric state

$$
B^{0} B^{0}+B^{0} \bar{B}^{0}+\bar{B}^{0} B^{0}+\bar{B}^{0} \bar{B}^{0}
$$

Such a state cannot be readily produced in practice, but in principle it is possible if there exists an interaction with $\Delta B=2$, such as the hypothetical superweak interaction.

To summarize, we have studied inclusive decay time distributions of coherent two-body states. We find that there is a set of orthonormality relations among decay time distributions of states that are pure and orthogonal to each other at $t=0$. Using this, we have shown that the naive incoherent sum of single particle decay time distributions does not always give the correct inclusive distribution, and extracted conditions for it to be the case. Such 
incoherent sum was found to be correct for any $B^{0} \bar{B}^{0}$ state regardless of the orbital angular momentum.

\section{ACKNOWLEDGMENTS}

I would like to thank Y. Azimov, R. Briere, I. Dunietz, S. Glashow, S. Pakvasa, for useful discussions, and R. Madrak for reading the manuscript. This work was supported by the Department of Energy Grant DE-FG02-91ER40654.

\section{APPENDIX:}

For $n=2$, the condition (2.12) reduces to

$$
\begin{aligned}
& \Re\left(G q^{\prime *} p^{\prime}\right) e^{-\gamma_{a} t}\left|a_{a f}\right|^{2}-\Re\left(G q^{*} p\right) e^{-\gamma_{b} t}\left|a_{b f}\right|^{2} \\
& +\Re\left[\left(G^{*} p^{\prime *} q-G q^{\prime *} p\right) e^{-\left(\gamma_{+}-i \delta m\right) t} a_{a f}^{*} a_{b f}\right]=0 .
\end{aligned}
$$

Since the three terms have different time dependences, each term should separately be zero. The decay amplitudes are in general non-zero (we are requiring that the naive inclusive distribution be correct independent of decay amplitudes); thus, the above condition leads to

$$
\left\{\begin{array}{rl}
\Re\left(G q^{* *} p^{\prime}\right) & =0 \\
\Re\left(G q^{*} p\right) & =0 \\
G^{*} p^{* *} q-G q^{*} p & =0
\end{array},\right.
$$

where the last condition is due to the fact that the term $e^{i \delta m t}$ samples all possible phases. Now we define

$$
G \stackrel{\text { def }}{=}|G| e^{i g}, \quad s \stackrel{\text { def }}{\equiv} q e^{i g}, \quad s^{\prime} \stackrel{\text { def }}{\equiv} q^{\prime} e^{i g} .
$$

Substituting this in (A4), and dividing each equation by $|s|^{2}$, one obtains

$$
\left\{\begin{aligned}
|G| \Re\left(\frac{p^{\prime}}{s^{\prime}}\right) & =0 \\
|G| \Re\left(\frac{p}{s}\right) & =0 \quad ; \\
|G|\left[\left(\frac{p^{\prime}}{s^{\prime}}\right)^{*}-\frac{p}{s}\right] & =0
\end{aligned}\right.
$$


namely,

$$
|G|=0, \quad \text { or } \quad\left\{\begin{array}{l}
\frac{p^{\prime}}{s^{\prime}}, \frac{p}{s^{*}}: \text { pure imaginary, and } \\
\left(\frac{p^{\prime}}{s^{\prime}}\right)^{*}=\frac{p}{s}
\end{array}\right.
$$

Thus, if $|G| \neq 0$, this leads to

$$
\frac{p^{\prime}}{-q^{\prime}}=\frac{p}{q},
$$

which means that $B_{a}$ and $B_{b}$ are same physical states and it contradicts our assumption that they have different decay rates and masses. Thus, $G=0$ results from (A1). On the other hand, the condition $G=0$ trivially leads to (A1); thus, $G=0$ is the necessary and sufficient condition for the naive inclusive distribution to be correct assuming that $\gamma_{a} \neq \gamma_{b}$, $m_{a} \neq m_{b}$ and $p, q, p^{\prime}, q^{\prime}$ are non-zero (namely, $B^{0}$ and $\bar{B}^{0} \operatorname{mix}$ ). 


\section{REFERENCES}

[1] See for example, C. D. Buchanan, R. Cousins, C. Dib, R.D. Peccei, and J. Quackenbush, Phys. Rev. D45, 4088 (1992); M. Hayakawa and A.I. Sanda, Phys. Rev. D48, 1150 (1993).

[2] Y. Nir and H.R. Quinn, in 'B Decays', 2nd ed., Ed. S. Stone, World Scientific, 1994, and references therein.

[3] H. Yamamoto, hep-ph/9703336, to be published in Phys. Lett. B.

[4] For example, if one assumes that $\operatorname{Amp}\left(B^{0} \rightarrow K^{-} \pi^{+}\right)=\operatorname{Amp}\left(\bar{B}^{0} \rightarrow K^{+} \pi^{-}\right)=0$, then in spite of $B^{0}-\bar{B}^{0}$ mixing or $C P$ violation, one can show that the inclusive yield ratio measured on $\Upsilon(4 S)$ is related to the amplitude ratio by $N\left(K^{-} \pi^{+}\right) / N\left(K^{+} \pi^{-}\right)=$ $\left|A m p\left(\bar{B}^{0} \rightarrow K^{-} \pi^{+}\right) / A m p\left(B^{0} \rightarrow K^{+} \pi^{-}\right)\right|^{2}$.

[5] V.F. Weisskopf and E.P. Wigner, Z. Phys. 63, 54 (1930); ibid., 65, 18 (1930).

[6] Typical deviations in decay distributions from the Weisskopf-Wigner approximation is of order $\delta m / m \ll 1$. For more discussions, see for example, P.K. Kabir and A. Pilafsis, Phys. Rev. A53, 66 (1996), and references therein.

[7] J.S. Bell, Theory of Weak Interactions in Les Houches Summer School on Theoretical Physics, Les Houches, France, 1965, ed. C. de Witt and M. Jacob.

[8] The relation can readily be obtained from $\sum_{f}|A m p(\phi \rightarrow f)|^{2}=-d / d t\langle\phi \mid \phi\rangle$, where $\phi$ is an arbitrary linear combination of $B_{i}$. J.S. Bell and J. Steinberger, in Proceedings of the Oxford International Conference on Elementary Particles, 1965.

[9] T.D. Lee, Particle Physics and Introduction to Field Theory, Harwood Academic Publishers, 1981

[10] I would like to thank Y. Azimov for pointing out this interpretation. 